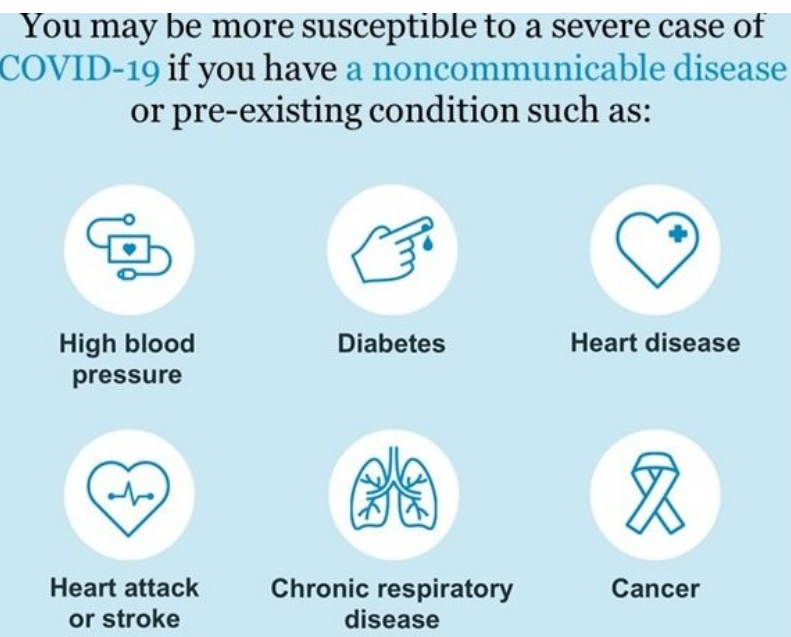

\title{
What Impact Have SARS-CoV-2/Covid-19 Pandemic induced lockdown on the number of OPD patients of Diabetes, Hypertension, Stroke (CVA), Acute Heart Disease, Mental IIIness, Epilepsy, Ophthalmic, Dental and oncology in India during the lockdown months (April/May/2020) Observational Research Analysis?
}

DR PIYUSH KUMAR

Funding: The author(s) received no specific funding for this work.

Potential competing interests: The author(s) declared that no potential competing interests exist.

Abstract

The First human case of the covid-19 global pandemic was reported from Wuhan city of China in December 2019. On 27th January 2020, India found a suspected case of covid-19 in Kerala with a recent travel history of Wuhan; china. The suspected case was reported as positive by the National Institute of Virology located at Pune in Maharashtra, India on January 30, 2020 as positive for COVID-19 infection. This was the first documented COVID-19 case in India. The government of India responded to this novel disease by enforcing nationwide lockdown starting on 25/03/2020 and ending on 31/05/2020. In this retrospective observational quantitative and qualitative study I have done assessment of the impact of covid-19 lockdown measures on OPD health services for some important non communicable diseases. The data is collected, observed, analyzed for the years 2020 and 2019 and the lockdown period of AprilMay 2020 is compared with the same duration of 2019 (excluding period of 7 days of lockdown in 
march) . The OPD (outpatient Department) services for the patients of Diabetes, Hypertension, Stroke (CVA), Acute Heart Disease, Mental IIIness, Epilepsy, Ophthalmic, Dental and oncology were selected for this observational retrospective study-analysis. In version 2 time series analysis (Ionger period) will be applied to test the significance of difference between pre and post intervention (lockdown). The study found as compared to 2019 when lockdown and pandemic was not in existence, 2020 lockdown period have notable reduction in various OPD health services. The study have derived conclusion that lockdown reduced number of OPD patients and hence reduced access to the health services for non-communicable disease which is the major burden of disease in India as well as at global levels. In the final conclusion the author feel the need of more emphasis and priority for NCDs care in any situations of emergency like pandemic and natural calamities, lockdowns etc which usually disrupt routine healthcare. Routine healthcare is very essential in context of chronic diseases which can be converted to acute emergency conditions like CVA.

\section{Keywords: OPD, Health facility, Covid-19, lockdown,}

\section{Background}

The SARS-CoV-2 virus was first reported from Wuhan City, Hubei province of China in December 2019 and has spread worldwide in more than 200 countries. The topmost health organization of the world (World Health Organization) WHO with reference and guidelines of the International Health Regulations (2005) declared COVID-19 outbreak a Public Health Emergency of International Concern on 30 January 2020 last year and started issuing various protocols, guidelines, advisories for all the nations of the world ${ }^{1}$. On 27 th January 2020, India found a suspected case of covid-19 in Kerala with a recent travel history of Wuhan; china. The suspected case was reported as positive by the National Institute of Virology located at Pune in Maharashtra, India on January 30, 2020 as positive for COVID-19 infection. This was the first documented COVID-19 case in India.

The government of India responded to this novel disease by enforcing nationwide lockdown starting on $25 / 03 / 2020$ and ending on 31/05/2020². Lockdown procedures, ensuring social distancing, and encouraging the populations to stay at home with hand hygiene and good ventilation with fresh air etc is being recommended by WHO time to time updated on daily basis almost ${ }^{3}$. Like most of the nations for saving lives India also imposed a strict lockdown countrywide. The lockdown helps to reduce numbers of new cases by putting a barrier in community spread while on the other hand it disrupted the routineregular functioning of the health system delivery as well as for LMICs (Low-middle-income countries) like India a massive economic impact is inevitable ${ }^{4}$.

The provision of health services is of prime importance and key concern in India especially because of high dense population load as well as fewer resources, old fashioned poor infrastructure and massive demand on healthcare system. The children's, senior citizens, and women's are especially vulnerable because of special requirements of health needs ${ }^{5}$. The ongoing with ups and downs, SARS-CoV-2 pandemic challenged healthcare systems around the world. There is a sense of fear around the whole world due to pandemic 
and India is not an exception. The limited healthcare facilities including infrastructure, transportation (ambulance services) etc. manpower, have been chiefly deployed to deal with the situation of Covid-19 pandemic. This shift has detrimental impact on ongoing various health services running previously before the pandemic era. Added to this there are other impacts on domestic violence etc which have shown an increasing trend against women ${ }^{6}$.

This observational retrospective study is highlighting that there is a very big global burden of disease called non-communicable disease particularly India is struggling with this NCD epidemic since several years. Through presentation of facts and figures the author hope that it will help policy and decision makers to think about measures of mitigation and providing relief in form of regular health services to NCDs patients. The research study specially highlights the use of novel intervention of lockdown and its impact on health service delivery and access to healthcare services. Any change in the health outcomes like numbers of OPD patients after application of lockdown intervention are assumed to be effects of this novel intervention. Except one week of March the whole period of lockdown will be compared to previous year of same timeline and duration to know the impact of intervention of lockdown as shown in title of research.

\section{Objectives of the study}

The key objectives of this retrospective observational analytic study are to evaluate the impact of the SARS-CoV-2 pandemic induced lockdown on OPD health service delivery in India. The prime objectives are to:

1. assess the increase/decrease in total number of OPD patients of Diabetes, Hypertension, Stroke (CVA), Acute Heart Disease, Mental IIIness, Epilepsy, Ophthalmic, Dental and oncology in India during the lockdown months (April-May-2020) and comparison along the timeline of previous year with no lockdown intervention of same period of all India

2. to assess the difference in rural and urban number of OPD patients of Diabetes, Hypertension, Stroke (CVA), Acute Heart Disease, Mental Illness, Epilepsy, Ophthalmic, Dental and oncology in India during the lockdown months (April-May-2020) and comparison along the timeline of previous year with no lockdown intervention of same period of all India

3. to assess the difference in public and private facilities number of OPD patients of Diabetes, Hypertension, Stroke (CVA), Acute Heart Disease, Mental Illness, Epilepsy, Ophthalmic, Dental and oncology in India during the lockdown months (April-May-2020) and comparison along the timeline of previous year with no lockdown intervention of same period of all India

In version $\mathbf{2}$ of this article hypothesis- instituting lockdown controlled the outbreak, but at the same time have devastating- detrimental consequences on health services and non health services will be tested and results will be displayed.

\section{Methods}

\section{Study method and sources of data}

This work is part of my retrospective observational study intended at describing the scenery, 
measurements, and range of the indirect health impacts of COVID-19 control measures particularly lockdown during the epidemic. I am using a case study approach for doing this work. This study will continue for a much longer time with focus to build evidence against or for a hypothesis. In this study I am focused on particular but several health effects and outcomes of response to answer objectives which will vary with versions of the study. The source of data is mainly the Health Management Information System (HMIS) of Ministry of Health and Family Welfare (MoHFW), Government of India for knowing the trends of outpatient department visits of Diabetes, Hypertension, Stroke (CVA), Acute Heart Disease, Mental Illness, Epilepsy, Ophthalmic, Dental and oncology in India during the lockdown months (April-May-2020), as affected by the covid-19 and also through Google search and personal sources which are accredited document/record/media review etc. to assess the pandemic impacts. The HMIS is a well established reporting system used by all the states and union territories of India available through MoHFW (the Ministry of Health India). The information on HMIS is uploaded on a routine basis from the entire health unit across the nation. Although I am having a very big data collection, in this article research I am discussing only about impact of covid-19 induced lockdown on number of OPD patients of Diabetes, Hypertension, Stroke (CVA), Acute Heart Disease, Mental IIIness, Epilepsy, Ophthalmic, Dental and oncology in India during the lockdown months (April-May-2020) compared to previous year same timeline and duration.

\section{Data analysis}

For the assessment of impact of covid-19 induced lockdown on number of OPD patients of Diabetes, Hypertension, Stroke (CVA), Acute Heart Disease, Mental Illness, Epilepsy, Ophthalmic, Dental and oncology in India during the lockdown months (April-May-2020) compared to previous year same timeline and duration, I plotted the monthly numbers of cases-total/public/private/rural/urban against time and compared with previous year. The version 2 of this article will have more periods of comparison and data of $\mathbf{3 6}$ states and union territories will be discussed in brief to reduce the length of

article. The National Disaster Management Authority (NDMA), Headed and chaired by Hon'ble Prime Minister of India Shri Narendra Modi, in exercise of the powers vested under section 6(2)(i) of the Disaster Management Act, 2005, has passed an Order dated 24.03.2020, directing the Ministries/ Departments of Government of India as well as the State/Union Territory Governments and State/ Union Territory concerned authorities to take proper and effective measures-protocols-guidelines to control the proliferation of COVID-19 in the nation.

Ministries/ Departments of Government of India as well as the State/Union Territory Governments and State/ Union Territory concerned authorities in compliance of the said Order of NDMA as well as order issued by Ministry of Home Affairs (MHA) dated 24.03.2020 under Section 10(2)(I) of the Disaster Management Act, directing the Ministries/ Departments of Government of India, State/Union Territory Governments and State/ Union Territory Authorities to take effective measures for ensuring social distancing so as to prevent the spread of COVID-19 in the country. The Order of lockdown remained in force, in all parts of the country, for a period of 21 days with effect from 25.03.2020 and extended further 
upto $31^{\text {st }}$ May. This fact is well documented in the newspapers, and international agencies like WHO and Government reports.

Data analysis presented in Table $1,2,3,4,5,6,7,8,9,10$ shows the list of OPD indicators and numbers of OPD used in the study for specific diseases mentioned in the title to know the intervention lockdown impacts of COVID-19 on health service delivery and utilization. Lockdown period is compared to same period of previous year. It's a well documented fact that population and NCD is increasing by leaps and bounds in India. Hence it is evident from the data analysis that the novel intervention of complete lockdown in India tends to have a detrimental and deleterious impact on health delivery services and utilization.

April-2019\& 2020

Table 1- showing total numbers of specific diseases OPD during April 2019 and 2020 and decrease in OPD during lockdown

\begin{tabular}{|c|c|c|c|c|}
\hline Services & $\begin{array}{l}\text { Total Apr- } \\
\text { 20(Number of } \\
\text { OPD) }\end{array}$ & $\begin{array}{l}\text { Total Apr- } \\
\text { 19(Number of } \\
\text { OPD) }\end{array}$ & $\begin{array}{l}\text { Total decrease in Apr- } 2020 \text { (Number of } \\
\text { OPD) in comparison to Apr-2019 }\end{array}$ & $\begin{array}{l}\text { Percent decrease in total OPD in Apr- } \\
2020 \text { in comparison to Apr-2019 }\end{array}$ \\
\hline $\begin{array}{l}\text { Outpatient - } \\
\text { Diabetes }\end{array}$ & 1644625 & 2644246 & -999621 & 37.803631 \\
\hline $\begin{array}{l}\text { Outpatient - } \\
\text { Hypertension }\end{array}$ & 2092962 & 3048900 & -955938 & 31.3535373 \\
\hline $\begin{array}{l}\text { Outpatient - } \\
\text { Stroke (Paralysis) }\end{array}$ & 32918 & 64075 & -31157 & 48.6258291 \\
\hline $\begin{array}{l}\text { Outpatient - Acute } \\
\text { Heart Diseases }\end{array}$ & 78852 & 237022 & -158170 & 66.7322021 \\
\hline $\begin{array}{l}\text { Outpatient - } \\
\text { Mental illness }\end{array}$ & 170345 & 486869 & -316524 & 65.0121491 \\
\hline $\begin{array}{l}\text { Outpatient - } \\
\text { Epilepsy }\end{array}$ & 42966 & 91340 & -48374 & 52.9603679 \\
\hline $\begin{array}{l}\text { Outpatient - } \\
\text { Ophthalmic } \\
\text { Related }\end{array}$ & 644210 & 2665999 & -2021789 & 75.836075 \\
\hline $\begin{array}{l}\text { Outpatient - } \\
\text { Dental }\end{array}$ & 678914 & 2335144 & -1656230 & 70.9262469 \\
\hline $\begin{array}{l}\text { Outpatient - } \\
\text { Oncology }\end{array}$ & 42566 & 167477 & -124911 & 74.5839727 \\
\hline
\end{tabular}

Table 2- showing total numbers of specific diseases OPD at Public Health facilities in India during April 2019 and 2020 and decrease in OPD during lockdown 


\begin{tabular}{|c|c|c|c|c|}
\hline Services & $\begin{array}{l}\text { Public Health } \\
\text { Facility Apr- } \\
\text { 20(Number of OPD) }\end{array}$ & $\begin{array}{l}\text { Public Health } \\
\text { Facility Apr- } \\
\text { 19(Number of OPD) }\end{array}$ & $\begin{array}{l}\text { Total decrease in Public Health Facility } \\
\text { Apr- } 2020 \text { (Number of OPD) in comparison } \\
\text { to Apr-2019 }\end{array}$ & $\begin{array}{l}\text { Percent decrease in public health } \\
\text { facility OPD in Apr-2020 in } \\
\text { comparison to Apr-2019 }\end{array}$ \\
\hline $\begin{array}{l}\text { Outpatient - } \\
\text { Diabetes }\end{array}$ & 1605927 & 2546368 & -940441 & 36.9326429 \\
\hline $\begin{array}{l}\text { Outpatient - } \\
\text { Hypertension }\end{array}$ & 2056021 & 2969055 & -913034 & 30.7516701 \\
\hline $\begin{array}{l}\text { Outpatient - } \\
\text { Stroke } \\
\text { (Paralysis) }\end{array}$ & 30350 & 59595 & -29245 & 49.0729088 \\
\hline $\begin{array}{l}\text { Outpatient - } \\
\text { Acute Heart } \\
\text { Diseases }\end{array}$ & 69002 & 206141 & -137139 & 66.5267948 \\
\hline $\begin{array}{l}\text { Outpatient - } \\
\text { Mental illness }\end{array}$ & 161080 & 449545 & -288465 & 64.1682145 \\
\hline $\begin{array}{l}\text { Outpatient - } \\
\text { Epilepsy }\end{array}$ & 41512 & 86650 & -45138 & 52.0923254 \\
\hline $\begin{array}{l}\text { Outpatient - } \\
\text { Ophthalmic } \\
\text { Related }\end{array}$ & 625829 & 2516472 & -1890643 & 75.1306989 \\
\hline $\begin{array}{l}\text { Outpatient - } \\
\text { Dental }\end{array}$ & 667478 & 2285057 & -1617579 & 70.7894376 \\
\hline $\begin{array}{l}\text { Outpatient - } \\
\text { Oncology }\end{array}$ & 33381 & 145540 & -112159 & 77.0640374 \\
\hline
\end{tabular}

Table 3- showing total numbers of specific diseases OPD at Private Health Facilities in India during April 2019 and 2020 and decrease in OPD during lockdown 


\begin{tabular}{|c|c|c|c|c|}
\hline Services & $\begin{array}{l}\text { Private Health } \\
\text { Facility Apr- } \\
\text { 20(Number of OPD) }\end{array}$ & $\begin{array}{l}\text { Private Health } \\
\text { Facility Apr- } \\
\text { 19(Number of OPD) }\end{array}$ & $\begin{array}{l}\text { Total decrease in private Health Facility } \\
\text { Apr- } 2020 \text { (Number of OPD) in comparison } \\
\text { to Apr-2019 }\end{array}$ & $\begin{array}{l}\text { Percent decrease in private health } \\
\text { facility OPD in Apr-2020 in } \\
\text { comparison to Apr-2019 }\end{array}$ \\
\hline $\begin{array}{l}\text { Outpatient - } \\
\text { Diabetes }\end{array}$ & 38698 & 97878 & -59180 & 60.463025 \\
\hline $\begin{array}{l}\text { Outpatient - } \\
\text { Hypertension }\end{array}$ & 36941 & 79845 & -42904 & 53.73411 \\
\hline $\begin{array}{l}\text { Outpatient - } \\
\text { Stroke } \\
\text { (Paralysis) }\end{array}$ & 2568 & 4480 & -1912 & 42.678571 \\
\hline $\begin{array}{l}\text { Outpatient - } \\
\text { Acute Heart } \\
\text { Diseases }\end{array}$ & 9850 & 30881 & -21031 & 68.103365 \\
\hline $\begin{array}{l}\text { Outpatient - } \\
\text { Mental illness }\end{array}$ & 9265 & 37324 & -28059 & 75.17683 \\
\hline $\begin{array}{l}\text { Outpatient - } \\
\text { Epilepsy }\end{array}$ & 1454 & 4690 & -3236 & 68.997868 \\
\hline $\begin{array}{l}\text { Outpatient - } \\
\text { Ophthalmic } \\
\text { Related }\end{array}$ & 18381 & 149527 & -131146 & 87.707237 \\
\hline $\begin{array}{l}\text { Outpatient - } \\
\text { Dental }\end{array}$ & 11436 & 50087 & -38651 & 77.167728 \\
\hline $\begin{array}{l}\text { Outpatient - } \\
\text { Oncology }\end{array}$ & 9185 & 21937 & -12752 & 58.1301 \\
\hline
\end{tabular}

Table 4- showing total numbers of specific diseases OPD at urban health facilities in India during April 2019 and 2020 and decrease in OPD during lockdown 


\begin{tabular}{|c|c|c|c|c|}
\hline Services & $\begin{array}{l}\text { Urban Health } \\
\text { Facility Apr- } \\
\text { 20(Number of OPD) }\end{array}$ & $\begin{array}{l}\text { Urban Health } \\
\text { Facility Apr- } \\
\text { 19(Number of OPD) }\end{array}$ & $\begin{array}{l}\text { Total decrease in urban Health Facility } \\
\text { Apr- } 2020 \text { (Number of OPD) in comparison } \\
\text { to Apr-2019 }\end{array}$ & $\begin{array}{l}\text { Percent decrease in urban health } \\
\text { facility OPD in Apr-2020 in } \\
\text { comparison to Apr-2019 }\end{array}$ \\
\hline $\begin{array}{l}\text { Outpatient - } \\
\text { Diabetes }\end{array}$ & 413624 & 703624 & -290000 & 41.2151945 \\
\hline $\begin{array}{l}\text { Outpatient - } \\
\text { Hypertension }\end{array}$ & 505659 & 769481 & -263822 & 34.2857069 \\
\hline $\begin{array}{l}\text { Outpatient - } \\
\text { Stroke } \\
\text { (Paralysis) }\end{array}$ & 7921 & 10233 & -2312 & 22.5935698 \\
\hline $\begin{array}{l}\text { Outpatient - } \\
\text { Acute Heart } \\
\text { Diseases }\end{array}$ & 13708 & 39127 & -25419 & 64.9653692 \\
\hline $\begin{array}{l}\text { Outpatient - } \\
\text { Mental illness }\end{array}$ & 33083 & 107230 & -74147 & 69.1476266 \\
\hline $\begin{array}{l}\text { Outpatient - } \\
\text { Epilepsy }\end{array}$ & 11913 & 20358 & -8445 & 41.4824639 \\
\hline $\begin{array}{l}\text { Outpatient - } \\
\text { Ophthalmic } \\
\text { Related }\end{array}$ & 99196 & 547754 & -448558 & 81.8904107 \\
\hline $\begin{array}{l}\text { Outpatient - } \\
\text { Dental }\end{array}$ & 123720 & 430124 & -306404 & 71.2362017 \\
\hline $\begin{array}{l}\text { Outpatient - } \\
\text { Oncology }\end{array}$ & 8326 & 43244 & -34918 & 80.7464619 \\
\hline
\end{tabular}

Table 5- showing total numbers of specific diseases OPD at rural health facilities of India during April 2019 and 2020 and decrease in OPD during lockdown 


\begin{tabular}{|c|c|c|c|c|}
\hline Services & $\begin{array}{l}\text { Rural Health Facility } \\
\text { Apr-20(Number of } \\
\text { OPD) }\end{array}$ & $\begin{array}{l}\text { Rural Health Facility } \\
\text { Apr-19(Number of } \\
\text { OPD) }\end{array}$ & $\begin{array}{l}\text { Total decrease in Rural Health Facility Apr- } \\
2020 \text { (Number of OPD) in comparison to Apr- } \\
2019\end{array}$ & $\begin{array}{l}\text { Percent decrease in rural opd in } \\
\text { Apr-2020 in comparison to Apr- } \\
2019\end{array}$ \\
\hline $\begin{array}{l}\text { Outpatient - } \\
\text { Diabetes }\end{array}$ & 1231001 & 1940622 & -709621 & 36.5666781 \\
\hline $\begin{array}{l}\text { Outpatient - } \\
\text { Hypertension }\end{array}$ & 1587303 & 2279419 & -692116 & 30.3637023 \\
\hline $\begin{array}{l}\text { Outpatient - } \\
\text { Stroke } \\
\text { (Paralysis) }\end{array}$ & 24997 & 53842 & -28845 & 53.5734185 \\
\hline $\begin{array}{l}\text { Outpatient - } \\
\text { Acute Heart } \\
\text { Diseases }\end{array}$ & 65144 & 197895 & -132751 & 67.0815331 \\
\hline $\begin{array}{l}\text { Outpatient - } \\
\text { Mental illness }\end{array}$ & 137262 & 379639 & -242377 & 63.8440729 \\
\hline $\begin{array}{l}\text { Outpatient - } \\
\text { Epilepsy }\end{array}$ & 31053 & 70982 & -39929 & 56.2522893 \\
\hline $\begin{array}{l}\text { Outpatient - } \\
\text { Ophthalmic } \\
\text { Related }\end{array}$ & 545014 & 2118245 & -1573231 & 74.2704928 \\
\hline $\begin{array}{l}\text { Outpatient - } \\
\text { Dental }\end{array}$ & 555194 & 1905020 & -1349826 & 70.856264 \\
\hline $\begin{array}{l}\text { Outpatient - } \\
\text { Oncology }\end{array}$ & 34240 & 124233 & -89993 & 72.438885 \\
\hline
\end{tabular}

May 2019 \& 2020

Table 6- showing total numbers of specific diseases OPD during May 2019 and 2020 and decrease in OPD during lockdown 


\begin{tabular}{|c|c|c|c|c|}
\hline Services & $\begin{array}{l}\text { Total May- } \\
\text { 20(Number of } \\
\text { OPD) }\end{array}$ & $\begin{array}{l}\text { Total May- } \\
\text { 19(Number of } \\
\text { OPD) }\end{array}$ & $\begin{array}{l}\text { Total decrease in May- } 2020 \text { (Number of } \\
\text { OPD) in comparison to May-2019 }\end{array}$ & $\begin{array}{l}\text { Percent decrease in total OPD in May- } \\
2020 \text { in comparison to May-2019 }\end{array}$ \\
\hline $\begin{array}{l}\text { Outpatient - } \\
\text { Diabetes }\end{array}$ & 1703871 & 2795140 & -1091269 & 39.041658 \\
\hline $\begin{array}{l}\text { Outpatient - } \\
\text { Hypertension }\end{array}$ & 2141928 & 3281803 & -1139875 & 34.7331939 \\
\hline $\begin{array}{l}\text { Outpatient - } \\
\text { Stroke (Paralysis) }\end{array}$ & 36011 & 67151 & -31140 & 46.3730994 \\
\hline $\begin{array}{l}\text { Outpatient - Acute } \\
\text { Heart Diseases }\end{array}$ & 97046 & 249823 & -152777 & 61.1540971 \\
\hline $\begin{array}{l}\text { Outpatient - } \\
\text { Mental illness }\end{array}$ & 197833 & 505997 & -308164 & 60.9023374 \\
\hline $\begin{array}{l}\text { Outpatient - } \\
\text { Epilepsy }\end{array}$ & 46798 & 88047 & -41249 & 46.8488421 \\
\hline $\begin{array}{l}\text { Outpatient - } \\
\text { Ophthalmic } \\
\text { Related }\end{array}$ & 815141 & 2734647 & -1919506 & 70.1920943 \\
\hline $\begin{array}{l}\text { Outpatient - } \\
\text { Dental }\end{array}$ & 791717 & 2536955 & -1745238 & 68.7926274 \\
\hline $\begin{array}{l}\text { Outpatient - } \\
\text { Oncology }\end{array}$ & 63196 & 169710 & -106514 & 62.7623593 \\
\hline
\end{tabular}

Table 7- showing total numbers of specific diseases OPD at Public Health facilities in India during May 2019 and 2020 and decrease in OPD during lockdown 


\begin{tabular}{|c|c|c|c|c|}
\hline Services & $\begin{array}{l}\text { Public Health } \\
\text { Facility May- } \\
\text { 20(Number of OPD) }\end{array}$ & $\begin{array}{l}\text { Public Health } \\
\text { Facility May- } \\
\text { 19(Number of OPD) }\end{array}$ & $\begin{array}{l}\text { Total decrease in Public Health Facility } \\
\text { May- 2020(Number of OPD) in } \\
\text { comparison to May- } 2019\end{array}$ & $\begin{array}{l}\text { Percent decrease in public health } \\
\text { facility OPD in May- } 2020 \text { in } \\
\text { comparison to May-2019 }\end{array}$ \\
\hline $\begin{array}{l}\text { Outpatient - } \\
\text { Diabetes }\end{array}$ & 1653763 & 2696173 & -1042410 & 38.662578 \\
\hline $\begin{array}{l}\text { Outpatient - } \\
\text { Hypertension }\end{array}$ & 2093739 & 3200349 & -1106610 & 34.577791 \\
\hline $\begin{array}{l}\text { Outpatient - } \\
\text { Stroke } \\
\text { (Paralysis) }\end{array}$ & 32336 & 61844 & -29508 & 47.713602 \\
\hline $\begin{array}{l}\text { Outpatient - } \\
\text { Acute Heart } \\
\text { Diseases }\end{array}$ & 81880 & 218110 & -136230 & 62.45931 \\
\hline $\begin{array}{l}\text { Outpatient - } \\
\text { Mental illness }\end{array}$ & 186264 & 468451 & -282187 & 60.238317 \\
\hline $\begin{array}{l}\text { Outpatient - } \\
\text { Epilepsy }\end{array}$ & 45268 & 83440 & -38172 & 45.747843 \\
\hline $\begin{array}{l}\text { Outpatient - } \\
\text { Ophthalmic } \\
\text { Related }\end{array}$ & 779471 & 2577502 & -1798031 & 69.758666 \\
\hline $\begin{array}{l}\text { Outpatient - } \\
\text { Dental }\end{array}$ & 773557 & 2433846 & -1660289 & 68.216683 \\
\hline $\begin{array}{l}\text { Outpatient - } \\
\text { Oncology }\end{array}$ & 44506 & 139843 & -95337 & 68.17431 \\
\hline
\end{tabular}

Table 8- showing total numbers of specific diseases OPD at Private Health Facilities in India during May 2019 and 2020 and decrease in OPD during lockdown 


\begin{tabular}{|c|c|c|c|c|}
\hline Services & $\begin{array}{l}\text { Private Health } \\
\text { Facility May- } \\
\text { 20(Number of OPD) }\end{array}$ & $\begin{array}{l}\text { Private Health } \\
\text { Facility May- } \\
\text { 19(Number of OPD) }\end{array}$ & $\begin{array}{l}\text { Total decrease in private Health Facility } \\
\text { May- } 2020 \text { (Number of OPD) in } \\
\text { comparison to May-2019 }\end{array}$ & $\begin{array}{l}\text { Percent decrease in private health } \\
\text { facility OPD in May- } 2020 \text { in } \\
\text { comparison to May- } 2019\end{array}$ \\
\hline $\begin{array}{l}\text { Outpatient - } \\
\text { Diabetes }\end{array}$ & 50108 & 98967 & -48859 & 49.3689816 \\
\hline $\begin{array}{l}\text { Outpatient - } \\
\text { Hypertension }\end{array}$ & 48189 & 81454 & -33265 & 40.8390012 \\
\hline $\begin{array}{l}\text { Outpatient - } \\
\text { Stroke } \\
\text { (Paralysis) }\end{array}$ & 3675 & 5307 & -1632 & 30.7518372 \\
\hline $\begin{array}{l}\text { Outpatient - } \\
\text { Acute Heart } \\
\text { Diseases }\end{array}$ & 15166 & 31713 & -16547 & 52.1773405 \\
\hline $\begin{array}{l}\text { Outpatient - } \\
\text { Mental illness }\end{array}$ & 11569 & 37546 & -25977 & 69.1871305 \\
\hline $\begin{array}{l}\text { Outpatient - } \\
\text { Epilepsy }\end{array}$ & 1530 & 4607 & -3077 & 66.7896679 \\
\hline $\begin{array}{l}\text { Outpatient - } \\
\text { Ophthalmic } \\
\text { Related }\end{array}$ & 35670 & 157145 & -121475 & 77.3012186 \\
\hline $\begin{array}{l}\text { Outpatient - } \\
\text { Dental }\end{array}$ & 18160 & 103109 & -84949 & 82.3875704 \\
\hline $\begin{array}{l}\text { Outpatient - } \\
\text { Oncology }\end{array}$ & 18690 & 29867 & -11177 & 37.4225734 \\
\hline
\end{tabular}

Table 9- showing total numbers of specific diseases OPD at urban health facilities in India during May 2019 and 2020 and decrease in OPD during lockdown 


\begin{tabular}{|c|c|c|c|c|}
\hline Services & $\begin{array}{l}\text { Urban Health Facility } \\
\text { May-20(Number of } \\
\text { OPD) }\end{array}$ & $\begin{array}{l}\text { Urban Health Facility } \\
\text { May-19(Number of } \\
\text { OPD) }\end{array}$ & $\begin{array}{l}\text { Total decrease in urban Health Facility May- } \\
2020 \text { (Number of OPD) in comparison to May- } \\
2019\end{array}$ & $\begin{array}{l}\text { Percent decrease in May- } \\
2020 \text { in comparison to May- } \\
2019\end{array}$ \\
\hline $\begin{array}{l}\text { Outpatient - } \\
\text { Diabetes }\end{array}$ & 409952 & 733674 & -323722 & 44.1234118 \\
\hline $\begin{array}{l}\text { Outpatient - } \\
\text { Hypertension }\end{array}$ & 488199 & 796315 & -308116 & 38.6927284 \\
\hline $\begin{array}{l}\text { Outpatient - } \\
\text { Stroke } \\
\text { (Paralysis) }\end{array}$ & 8895 & 13029 & -4134 & 31.7292194 \\
\hline $\begin{array}{l}\text { Outpatient - } \\
\text { Acute Heart } \\
\text { Diseases }\end{array}$ & 16176 & 41360 & -25184 & 60.8897485 \\
\hline $\begin{array}{l}\text { Outpatient - } \\
\text { Mental illness }\end{array}$ & 48147 & 110314 & -62167 & 56.3545878 \\
\hline $\begin{array}{l}\text { Outpatient - } \\
\text { Epilepsy }\end{array}$ & 12489 & 20025 & -7536 & 37.6329588 \\
\hline $\begin{array}{l}\text { Outpatient - } \\
\text { Ophthalmic } \\
\text { Related }\end{array}$ & 128354 & 522125 & -393771 & 75.4169978 \\
\hline $\begin{array}{l}\text { Outpatient - } \\
\text { Dental }\end{array}$ & 142821 & 463955 & -321134 & 69.2166266 \\
\hline $\begin{array}{l}\text { Outpatient - } \\
\text { Oncology }\end{array}$ & 13111 & 33255 & -20144 & 60.5743497 \\
\hline
\end{tabular}

Table 10- showing total numbers of specific diseases OPD at rural health facilities of India during May 2019 and 2020 and decrease in OPD during lockdown 


\begin{tabular}{|c|c|c|c|c|}
\hline Services & $\begin{array}{l}\text { Rural Health Facility } \\
\text { May-20(Number of } \\
\text { OPD) }\end{array}$ & $\begin{array}{l}\text { Rural Health Facility } \\
\text { May-19(Number of } \\
\text { OPD) }\end{array}$ & $\begin{array}{l}\text { Total decrease in Rural Health Facility May- } \\
2020 \text { (Number of OPD) in comparison to May- } \\
2019\end{array}$ & $\begin{array}{l}\text { Percent decrease in May- } \\
2020 \text { in comparison to May- } \\
2019\end{array}$ \\
\hline $\begin{array}{l}\text { Outpatient - } \\
\text { Diabetes }\end{array}$ & 1293919 & 2061466 & -767547 & 37.23307 \\
\hline $\begin{array}{l}\text { Outpatient - } \\
\text { Hypertension }\end{array}$ & 1653729 & 2485488 & -831759 & 33.46462 \\
\hline $\begin{array}{l}\text { Outpatient - } \\
\text { Stroke } \\
\text { (Paralysis) }\end{array}$ & 27116 & 54122 & -27006 & 49.89838 \\
\hline $\begin{array}{l}\text { Outpatient - } \\
\text { Acute Heart } \\
\text { Diseases }\end{array}$ & 80870 & 208463 & -127593 & 61.20655 \\
\hline $\begin{array}{l}\text { Outpatient - } \\
\text { Mental illness }\end{array}$ & 149686 & 395683 & -245997 & 62.17022 \\
\hline $\begin{array}{l}\text { Outpatient - } \\
\text { Epilepsy }\end{array}$ & 34309 & 68022 & -33713 & 49.56191 \\
\hline $\begin{array}{l}\text { Outpatient - } \\
\text { Ophthalmic } \\
\text { Related }\end{array}$ & 686787 & 2212522 & -1525735 & 68.95909 \\
\hline $\begin{array}{l}\text { Outpatient - } \\
\text { Dental }\end{array}$ & 648896 & 2073000 & -1424104 & 68.69773 \\
\hline $\begin{array}{l}\text { Outpatient - } \\
\text { Oncology }\end{array}$ & 50085 & 136455 & -86370 & 63.29559 \\
\hline
\end{tabular}

In version 2 of the article which is under process, the Interrupted time series (ITS) analysis will be done which is a strong quasi-experimental design and utilized to judge the effectiveness of a population-level based intervention such as Lockdown.

\section{Ethics approval for the study}

The data was made available from the Ministry of Health and Family Welfare. Being a Government servant of Health department author consulted some officials. The study have not involved any human being or animal in real and no trial etc. was done by the author hence this study didn't required any approvals from the concerned body or departments.

\section{Results}

\section{Secondary impacts of COVID-19 induced lockdown on health services:}

The lockdown imposition controlled the outbreak at the cost of health service systems and livelihoods disruption. The government, diverted health personnel and resources away from priority NCD and other services like immunization, ANC, Family Planning, domestic violence, mental health etc. resulting in deterioration of essential health service utilization and delivery $5,6,7,8,9$. The impact on patients of chronic conditions such as NCDs who need regular check-up and medicines for better health and good quality of life were unable to reach at OPD as evident from this retrospective observational study. India being a poor country, it is expected that most of the population below poverty line could not afford medication during the lockdown which is provided free at public health facilities 
Patients with newly diagnosed NCDs may not able to get the treatment, while the old chronic NCDs patients may have missed their regular therapy. It is quite evident from this study that majority of patients with title mentioned conditions faced an increased risk of complications, morbidity and mortality due to inability to access healthcare because of novel intervention of lockdown causing fear of contracting the virus from healthcare facilities/personals, transport restrictions, curfew, etc. It's also a well documented fact that delayed initiations and interruption of treatment regimens may increase disease progression, recurrence, stress, anxiety, and premature mortality with morbidity.

\section{Access to health service and utilization}

The total OPD of all diseases in this study have shown a drastic reduction in numbers as well as in percentages of OPD during lockdown intervention period as shown in table 1and 6 and annexure 1, 2 for the month of April and May respectively.

The total number of diabetes patients accessing health facilities declined by -999621 numbers and $37.803631 \%$ as compared to previous year April 2019 OPD. The total number of diabetes patients accessing health facilities declined by $-1091269,39.041658$ numbers and percent respectively as compared to previous year May 2019 OPD. The total number of hypertension patients accessing health facilities declined by $-955938,31.3535373$ numbers and percent respectively as compared to previous year April 2019 OPD. The total number of hypertension patients accessing health facilities declined by 1139875, 34.7331939numbers and percent respectively as compared to previous year May 2019 OPD. The total number of Stroke (Paralysis) patients accessing health facilities declined by -31157 , 48.6258291numbers and percent respectively as compared to previous year April 2019 OPD. The total number of Stroke (Paralysis) patients accessing health facilities declined by -31140, 46.3730994 numbers and percent respectively as compared to previous year May 2019 OPD. The total number of Acute Heart Diseases patients accessing health facilities declined by $-158170,66.7322021$ numbers and percent respectively as compared to previous year April 2019 OPD. The total number of Acute Heart Diseases patients accessing health facilities declined by $-152777,61.1540971$ numbers and percent respectively as compared to previous year May 2019 OPD. The total number of mental illness patients accessing health facilities declined by $-316524,65.0121491$ numbers and percent respectively as compared to previous year April 2019 OPD. The total number of mental illness patients accessing health facilities declined by $308164,60.9023374$ numbers and percent respectively as compared to previous year May 2019 OPD. The total number of epilepsy patients accessing health facilities declined by $-48374,52.9603679$ numbers and percent respectively as compared to previous year April 2019 OPD. The total number of epilepsy patients accessing health facilities declined by $-41249,46.8488421$ numbers and percent respectively as compared to previous year May 2019 OPD. The total number of eye patients, accessing health facilities declined by 2021789, 75.836075 numbers and percent respectively as compared to previous year April 2019 OPD. The total number of eye patients accessing health facilities declined by -1919506, 70.1920943 numbers and percent respectively as compared to previous year May 2019 OPD. The total number of dental opd patients, accessing health facilities declined by $-1656230,70.9262469$ numbers and percent respectively 
as compared to previous year April 2019 OPD. The total number of dental opd patients accessing health facilities declined by $-1745238,68.7926274$ numbers and percent respectively as compared to previous year May 2019 OPD. The total number of oncology opd patients, accessing health facilities declined by $124911,74.5839727$ numbers and percent respectively as compared to previous year April 2019 OPD. The total number of oncology opd patients accessing health facilities declined by $-106514,62.7623593$ numbers and percent respectively as compared to previous year May 2019 OPD.

The total public health facility OPD of all diseases in this study have shown a drastic reduction in numbers as well as in percentages of OPD during lockdown intervention period as shown in table 2and 7 and annexure 3, 4 for the month of April and May respectively. All the results are not described here in sentences as it is well presented in the form of tables 1 to 10 and annexure $1,2,3,4,5,6,7,8,9,10$ to reduce the length and words of this article.

\section{Discussion}

As mentioned above, the results of this study found that the complete lockdown in India during April, and May-2020 had negatively affected access and utilization of health services of OPD of title mentioned diseases. This negative impact of lockdown intervention is due to many factors, such as health workers being shifted for controlling the COVID-19 pandemic and therefore not available for other healthcare services. The number of OPD declined may be explained by a possibility that prior knowledge of COVID-19 through media and communication channels might have influenced prior health-seeking behavior. The government is forced by external and internal pressure to impose strict restrictions due to novel nature of covid-19 which is still under study. Lockdowns are not the best choice for countries like India, and other LMICs with a huge population.

\section{Limitations of this study}

The key data source for this study is HMIS, MoHFW. In this covid-19 pandemic, proper data collection is a big task and questionable. HMIS does data refreshment sometimes and personal collection of such big data is impossible and thus further analysis is limited. A very important limitation is that health awareness and related communication against COVID-19 started earlier than lockdown period. Hence very cautious people may have stopped visiting healthcare centers as a preventive action against COVID-19. The lockdown intervention was implemented throughout the nation leading to no areas that can be taken as control.

\section{Conclusions and recommendations derived from this study}

This retrospective observational quantitative and qualitative research study came to conclusion that the lockdown management to reduce or control COVID-19 had a massive negative impact on delivery and utilization of important/essential health services, and this aspect is clearly evident in April-May 2020. In the light of findings of this study I recommend the following:

- Governments of India should think of ways and strategies on priority basis to reduce the burden as well as morbidity and mortality occurring from NCDs. Compared to COVID-19 cases and deaths there are 
several other diseases which can obstruct India's way to reap the benefits of Demographic Dividend. The COVID-19 management should be prioritized, along with proper management of Other NCDs and CDs otherwise there are chances of increase in morbidity and mortality from several other diseases.

- Government should respond to COVID-19 with due considerations of stringent restrictions which can disrupt essential health services, leading to a vicious cycle and devastating effect on the health of population and economic crisis. Government should think of alternatives such as implementing standard operating procedures rather than imposing a lockdown.

- Government of India should give more focus on data collection system with inputs from local communities. There could have been more persons in the communities such as new NCDs cases who have not reached public/private health facilities which are not reflected in the HMIS data.

\section{'Declarations':}

-This paper has not been previously published and is not currently under consideration by another journal. The document is Microsoft word with English (United States) language \& 5350 words Total.

- Ethics approval and consent to participate: Not applicable. This study has not involved any human or animals in real or for experiments. The data on the prevalence and mortality due to COVID-19 pandemic were taken from the Health Department, Bihar and other organizations which are also available online, hence ethical approval was not required.

-Consent for publication: Not applicable

-Availability of data and materials: The data \& materials for study are mentioned in article and available as reference.

-Conflicts of Interest/ Competing Interest: There are no conflicts / competing of interest

- Funding-Self sponsored. No aid taken from individual or agency etc.

- Authors' contributions: The whole work is solely done and verified by the Author - Dr Piyush Kumar, M.B.B.S. - Sri Krishna Medical College, EMOC- General Medical Officer- Bihar Health Services- Government of Bihar, India.

- Acknowledgements- I am thankful to Advocate Anupama my wife for cooperation.

- Author information: The author is currently working as general medical officer for the government of Bihar.

-Financial Support \& sponsorship: Nil

\section{-Author contact information}

1 Department of Health, Government of Bihar, MOBILE - +919955301119/+917677833752,

Email drpiyush003@gmail.com

\section{References:}

1. Kumar, Piyush, What Are the Factors Responsible for Increase in SARS-CoV-2/COVID-19 Pandemic Related Cases and Death in India in 2021? How Does Environmental, Host \& Agent Factors of Epidemiological Triad Do Influence \& Can Be Utilised to Manage Ongoing Pandemic Cases and Deaths? 
(April 25, 2021). Available at SSRN: https://ssrn.com/abstract=3833788 or http://dx.doi.org/10.2139/ssrn.3833788

2. Andrews MA, Areekal B, Rajesh KR, Krishnan J, Suryakala R, Krishnan B, Muraly CP, Santhosh PV. First confirmed case of COVID-19 infection in India: A case report. Indian J Med Res. 2020 May;151(5):490492. doi: 10.4103/ijmr.IJMR_2131_20. PMID: 32611918; PMCID: PMC7530459.

3. Kumar, Piyush, Precaution \& Safety Methods for Hospital Duty During COVID-19 Pandemic Era for Healthcare Workers of Bihar, India: A Protective \& Supportive Approach (May 26, 2021). Available at SSRN: https://ssrn.com/abstract $=3842580$

4. Kumar, Piyush, What Impact Has Lockdown on SARS-CoV-2/COVID-19 Incidence, Prevalence and Mortality During Second Wave of Pandemic in 2021: Observational Analysis of Bihar (6/20/2021). Available at SSRN: https://ssrn.com/abstract=3871321 or http://dx.doi.org/10.2139/ssrn.3871321

5. DR PIYUSH KUMAR. What Impact Have SARS-CoV-2/Covid-19 Pandemic on the Reproductive and Child Health Programme of India over the 3 months after nationwide Lock down announcement in March 2020 -A brief analysis., 25 March 2021, PREPRINT (Version 1) available at Research Square [https://doi.org/10.21203/rs.3.rs-360551/v1]

6. Kumar, Piyush, What Impact Have SARS-CoV-2/COVID-19 Pandemic on Domestic Violence against Women in India across Different States and Union Territories from the Beginning of Lockdown Due to COVID-19 Pandemic in March 2020 Till 20Th September 2020? How COVID-19 Pandemic Induced Lockdown Influence Mental Health of Women in India? (April 15, 2021). Available at SSRN: https://ssrn.com/abstract=3826837 or http://dx.doi.org/10.2139/ssrn.3826837

7. Piyush Kumar. What Impact Have SARS-CoV-2/Covid-19 Pandemic on the Reproductive and Child Health Programme of Uttar Pradesh in India over the 3 months after nationwide Lockdown announcement in March 2020 -A brief analysis, 25 March 2021, PREPRINT (Version 1) available at Research Square [https://doi.org/10.21203/rs.3.rs-350287/v1]

8. Dr. Piyush Kumar. What Impact Have SARS-CoV-2/Covid-19 Pandemic on the Reproductive and Child Health Programme of Bihar in India over the 3 months after nationwide Lock down announcement in March 2020? How SARS-CoV-2 Pandemic era does influence RCH Programme? Immunisation? \&nbsp;Maternal Health? Family Planning?, 30 March 2021, PREPRINT (Version 4) available at Research Square [https://doi.org/10.21203/rs.3.rs-348841/v4]

9. Immunisation? Maternal Health? Family Planning?. https://doi.org/10.31219/osf.io/dphgaØKumar, P. (2021, March 28). What Impact Have SARS-CoV-2/Covid-19 Pandemic on the Reproductive and Child Health Programme (Immunisation, Maternal Health, Family Planning) of Andhra Pradesh in India over the 3 months after nationwide Lock down announcement in March 2020?

Annexure-1- comparison of total numbers of specific diseases OPD during April -2019 and 2020 - see table 1 
Annexure-2- comparison of total numbers of specific diseases OPD during May-2019 and 2020- see-table-6

Annexure-3- Comparison of total numbers of specific diseases OPD at Public Health facilities in India during April 2019 and 2020 - see table-2

Annexure-4- Comparison of total numbers of specific diseases OPD at Public Health facilities in India during May 2019 and 2020 - see table-7

Annexure-5- Comparison of total numbers of specific diseases OPD at private Health facilities in India during April 2019 and 2020 - see table-3

Annexure-6- Comparison of total numbers of specific diseases OPD at private Health facilities in India during May 2019 and 2020 - see table-8

Annexure-7- Comparison of total numbers of specific diseases OPD at Urban Health facilities in India during April 2019 and 2020 - see table-4

Annexure-8- Comparison of total numbers of specific diseases OPD at Urban Health facilities in India during May 2019 and 2020 - see table-9

Annexure-9- Comparison of total numbers of specific diseases OPD at Rural Health facilities in India during 
April 2019 and 2020 - see table-5

Annexure-10- Comparison of total numbers of specific diseases OPD at Rural Health facilities in India during May 2019 and 2020 - see table-10 